\title{
Índice de función sexual en mujeres que ejercen el comercio sexual
}

\author{
María Ruth Del Valle ${ }^{1 a}$, María Teresa Silva ${ }^{1 b}$, Gloria Venegas ${ }^{2 c}$, Rodrigo Gatica. ${ }^{1 d}$ \\ ${ }^{1}$ Facultad de Medicina, Universidad San Sebastián, Puerto Montt, Chile. ${ }^{2}$ Unidad de Atención y Control en Salud Sexual \\ (UNACESS), Hospital Las Higueras de Talcahuano, Chile.
}

$\mathrm{a}_{\text {Matrona. }}{ }^{\mathrm{b}}$ Estadístico, MSc. ${ }^{\mathrm{c}}$ Matrona, MSc. ${ }^{\mathrm{d}}$ Veterinario, PhD.

\section{SUMARIO}

Antecedentes: La sexualidad se muestra como un reflejo del nivel de bienestar físico, psicológico y social. Por lo tanto, las experiencias sexuales negativas pueden afectar el completo desarrollo como seres humanos. En Chile no existen estudios que indaguen en la presencia de disfunciones sexuales en la trabajadora sexual. Objetivo: El objetivo de este estudio es evaluar el Índice de Función Sexual Femenina (IFSF) en mujeres trabajadoras sexuales con respecto a su desempeño sexual con su pareja estable y compararlas con mujeres no trabajadoras sexuales. Método: Mujeres sexualmente activas mayores de 18 años. La toma de la muestra fue dirigida no probabilística intencional. El número final a estudiar fue de 58 mujeres, de las cuales 23 mujeres fueron trabajadoras sexuales (grupo estudio) y 35 mujeres que no se dedicaban al comercio sexual (grupo control). Resultados: La edad promedio fue 33 años para el grupo control y de 35 años en el caso de las trabajadoras sexuales. El $4 \%$ de las trabajadoras sexuales presentaba estudios universitarios, el $70 \%$ se encontraban solteras y utilizaron como principal método anticonceptivo el dispositivo intrauterino. En tanto, el 34\% del grupo control presentaba estudios universitarios, el $57 \%$ se encontraban solteras y utilizaron como principal método anticonceptivo el hormonal. No hubo diferencias significativas en el IFSF en general y por dominios, entre los grupos. Conclusiones: A pesar de presentar varios factores que aumentan el riesgo de disfunción sexual, las mujeres que desempeñan el comercio sexual no exhiben diferencias significativas en el IFSF respecto al grupo control.

\section{PALABRAS CLAVE: Sexualidad, trabajadoras sexual, mujer}

\section{SUMMARY}

Background: Sexuality is displayed as a reflection of the level of physical, psychological and social wellbeing. Therefore, negative sexual experiences can affect the entire development as human beings. In Chile, there are no studies that investigate the presence of sexual dysfunctions in the sex worker. Aim: To evaluate the Female Sexual Function Index (FSFI) in women sex workers about their sexual performance with his regular partner and compared with women not sex workers. Methods: Sexually active women over 18 years. We work with purposive intentional non probabilistic sampling. The final number of women studied was 58, of which 23 women were sex workers (study group) and 35 women were not involved in the sex trade (control group). Results: The average age was 33 years for the control group and 35 years in the case of sex workers. $4 \%$ of sex workers had university education, $70 \%$ were unmarried and used as main contraceptive the IUD. Meanwhile, $34 \%$ of the control group had university education, $57 \%$ were single and used as primary contraceptive the hormone method. There were no significant differences in overall 
IFSF and domains, between groups. Conclusions: Despite presenting several factors that increase the risk of sexual dysfunction, women played the sex trade do not exhibit significant differences in the FSFI with respect to the control group.

\section{KEY WORDS: Sexuality, sex workers, female}

\section{INTRODUCCIÓN}

La sexualidad es una parte integral de cada ser humano y es reflejo del bienestar físico, psicológico y social. Su desarrollo pleno depende de la satisfacción de necesidades humanas básicas como el deseo de contacto, intimidad, expresión emocional, placer, ternura y amor (1). La sexualidad desde la antigüedad era una necesidad imperante para saciar un impulso biológico que llevaba a la cópula, con dos objetivos principales: la reproducción y la mitigación de conflictos propios de la especie (2). Estudios en primates han determinado que la resolución de conflictos de forma no agresiva es más eficiente que el comportamiento agresivo y dentro de los primates, los bonobos, presentan las tasas más bajas de agresividad, generando la resolución de conflictos mediante el contacto genital (3).

Durante siglos la mujer fue menospreciada, postergada para cumplir el rol hija, esposa sumisa y madre abnegada, reprimiendo la expresión de su sexualidad por vergüenza y temor, ya que actuaba en función de lo que el hombre requería (4). La sexualidad de la mujer es el reflejo de su bienestar en diferentes niveles, tanto físico, psicológico como social. Sin embargo, la sociedad moderna ha restringido la sexualidad femenina y postergado la maternidad (5). Para la mayoría de las mujeres, la sexualidad continúa siendo un tema del que no se puede hablar libremente, junto con esto, el desconocimiento acerca de su propio cuerpo, funciones, sensaciones, emociones, necesidades y derechos, las lleva a adoptar un papel pasivo y de sometimiento, en el que las experiencias sexuales negativas pueden afectar en forma muy importante su desarrollo como seres humanos $(2,6)$.

Es así que la respuesta sexual fue descrita por primera vez en 1967 por William H. Masters y Virginia E. Johnson en el libro Respuesta Sexual Humana (7). Esta respuesta se entiende como el modo que cada persona tiene de reaccionar y responder al estímulo sexual, tanto de una manera física como desde el punto de vista más personal. Siendo, la salud sexual y reproductiva fundamental para las personas a lo largo de su ciclo vital, y sus consecuencias persisten en el período postreproductivo (8). Desde las descripciones del ciclo de respuesta sexual por Master y Johnson, se han producido sustanciales avances en el entendimiento de los aspectos fisiológicos de la función sexual (9). El orgasmo es una función psico-fisiológica normal del cuerpo humano y durante la respuesta sexual femenina, se producen cambios en las estructuras congestivas que son esenciales para el entendimiento de su respuesta sexual y específicamente del orgasmo (10). Esta respuesta está compuesta de cuatro elementos que se manifiestan de distintas maneras según el sexo, siendo estos: la excitación, meseta, el orgasmo y la resolución (1).

El estudio de la sexología se inició en Alemania a comienzos del siglo $X X$, con la fundación del instituto de sexología en la Universidad de Humboldt en Berlín (1919), realizándose un par de años después el primer congreso de sexología. Luego de la destrucción de este Instituto por los nazis en 1933, el campo de la sexología fue revitalizado por Alfred Kinsey, quien fundó el Instituto Kinsey para la investigación sexual en 1947 (1). En la década de los años 70 se comienza con un nuevo enfoque en la medicina sexual y el trabajo de Masters y Johnson fue sin duda, cada vez más conocido (11). En Chile, no existen estudios que indaguen en la presencia de disfunciones sexuales en la trabajadora sexual, ni mucho menos comparándola con mujeres que no se dedican a esta actividad, por lo tanto, esta investigación se presenta como una oportunidad para conocer la función sexual de la mujer y particularmente de la trabajadora sexual con pareja sentimentalmente comprometida.

El objetivo de este estudio es evaluar el Índice de Función Sexual Femenina (IFSF) en mujeres trabajadoras sexuales comparadas con mujeres no trabajadoras sexuales.

\section{SUJETOS Y METODOS}

Pacientes. La muestra fue tomada de mujeres controladas en consultorio de atención primaria CESFAM La Floresta de Hualpén y en Hospital Las Higueras de la Octava Región, Chile. La población incluida en la investigación corresponde a mujeres sexualmente activas mayores de 18 años. La toma de la muestra fue dirigida no probabilística intencional. El número final a estudiar fue de 58 mujeres, de las cuales 23 mujeres fueron trabajadoras sexuales (grupo estudio) y 35 mujeres que no se 
dedicaban al comercio sexual (grupo control). Las encuestadoras fueron 2 matronas que desempeñaban funciones en ambos centros asistenciales. Cada encuestada procedió a leer y contestar en forma personal las preguntas, dicha encuesta se llevó en dependencias de los centros de salud citados anteriormente, entre abril y septiembre del año 2011. Criterios de inclusión: mujeres mayores de 18 años que hayan tenido pareja sexual estable y cuyo estado de salud le permitiera desempeñar las actividades rutinarias. Criterios de exclusión: mujeres con discapacidad, patologías siquiátricas diagnosticadas y en tratamiento, histerectomizadas, con terapia de reemplazo hormonal (THR), embarazadas, puérperas, menopáusicas, que no hayan tenido pareja sexual estable. A las mujeres quienes cumplieron con los criterios de inclusión establecidos, se le informó que la consulta no tenía sólo carácter asistencial sino también investigativo, por lo tanto se les pidió su consentimiento de participación en el estudio.

Diseño. El diseño de esta investigación corresponde a un estudio de tipo transversal, descriptivo y comparativo entre mujeres que acuden a control en salud sexual en la UNACESS del Hospital las Higueras de Talcahuano (trabajadoras sexuales) y mujeres que se controlan en CESFAM La Floresta de Hualpén. En este estudio se evalúo la sexualidad femenina mediante la encuesta de Índice de Función Sexual Femenina (IFSF), con respecto a su desempeño sexual con su pareja estable. Este cuestionario ha sido validado por Rosen y cols (12) y en español por Blümel y cols (13). El cuestionario consta de dos partes, una evalúa los datos sociodemográficos de la usuaria y otro el IFSF de Rosen (12). Este último consiste en 19 preguntas agrupadas en seis dominios: deseo, excitación, lubricación, orgasmo, satisfacción y dolor. Cada pregunta tiene 5 ó 6 alternativas de respuesta, con un puntaje que varía desde 0 a 5 en cada pregunta. El puntaje total obtenido en la encuesta se obtiene mediante la suma aritmética de los productos obtenidos al multiplicar el promedio de cada dimensión por un factor. A mayor puntaje, aumenta la influencia positiva en la función sexual (13). Se aseguró la confidencialidad de la información recopilada la cual se ingresó sin nombre ni registro que pueda identificarlas.

Análisis estadístico. El análisis de los datos se

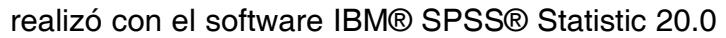
SPSS, y Microsoft Office Professional Pluss 2013. Se aplicó estadística descriptiva (promedios, desviación estándar, coeficiente de variación, máximo, mínimo y proporciones). Se utilizó gráficos de cajas y barras para comparación de grupos. La prueba
Mann Whitney permitió evaluar la diferencia de medias para grupos independiente y la Prueba $Z$ para diferencia de proporciones, considerando el supuesto $\mathrm{np}>5$. La no normalidad fue evaluada con test de Shapiro-Wilk. Se consideró significativo un valor $p \leq 0,05$.

\section{RESULTADOS}

De las 58 mujeres entrevistadas, 35 (60,3\%) correspondían al grupo control cuya edad promedio fue 32,8 \pm 8,1 años (rango: 22-54 años) y $23(39,7 \%)$ pertenecían al grupo de trabajadoras sexuales con una edad promedio de 35,6 \pm 8,8 años (rango: 21-45 años) (Figura 1).

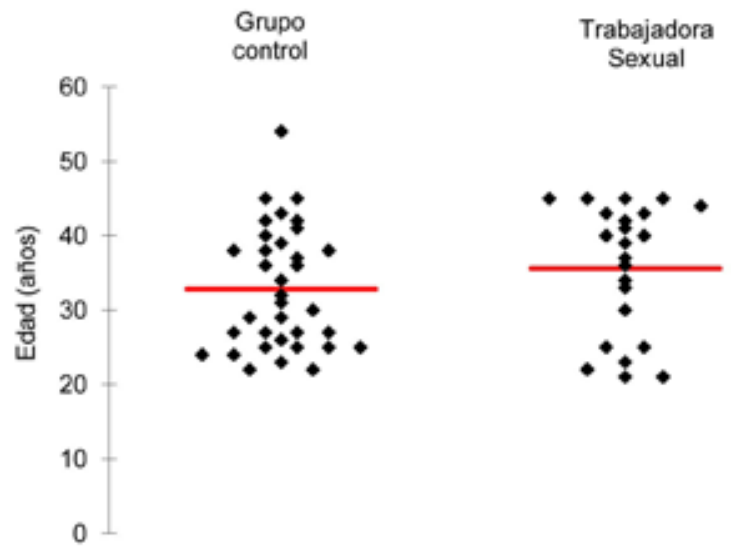

Figura 1. Distribución etaria del grupo control y grupo de trabajadoras sexuales

Respecto del nivel educacional el $34,3 \%$ de las mujeres del grupo control señaló haber cursado estudios superiores y solo el $4,3 \%(n=1)$ de las trabajadoras sexuales. En términos de educación media el porcentaje de mujeres en cada una de los grupos no presentó diferencias estadísticamente significativas (Tabla I).

Al analizar el estado civil se constató que el $69,6 \%$ de las trabajadoras sexuales eran solteras por sobre el $48,6 \%$ del grupo control, diferencia estadísticamente significativa $(p=0,05)$; esta situación civil fue la de mayor frecuencia en ambos grupos. Comparativamente, en el grupo control la segunda mayor frecuencia fue de mujeres casadas $42,9 \%$ y en el grupo de trabajadoras sexuales fue la convivencia con $26,1 \%$ (Tabla II) 
Tabla I

NÚMERO Y PORCENTAJE DE MUJERES POR GRUPOS SEGÚN NIVEL EDUCACIONAL

\begin{tabular}{ccccccc}
\hline Nivel educacional & \multicolumn{2}{c}{ Grupo control } & \multicolumn{2}{c}{ Trabajadora Sexual } & & $\mathrm{Z}_{\mathrm{C}}$ \\
\cline { 2 - 6 } & $\mathrm{n}$ & $\%$ & $\mathrm{n}$ & $\%$ & - & - \\
\hline Básica & 1 & 2,9 & 8 & 34,8 & 0,152 & 0,439 \\
Media & 22 & 62,9 & 14 & 60,9 & - & - \\
Superior & 12 & 34,3 & 1 & 4,3 & \\
\hline Total & 35 & 100,0 & 23 & 100,0 & \\
\hline
\end{tabular}

Tabla II

NÚMERO Y PORCENTAJE DE MUJERES POR GRUPO SEGÚN ESTADO CIVIL

\begin{tabular}{ccccccc}
\hline \multirow{2}{*}{ Estado civil } & \multicolumn{2}{c}{ Grupo control } & \multicolumn{2}{c}{ Trabajadora Sexual } & $\mathrm{Z}_{\mathrm{C}}$ & $\mathrm{p}$ \\
\cline { 2 - 7 } & $\mathrm{n}$ & $\%$ & $\mathrm{n}$ & $\%$ & & \\
\hline Soltera & 17 & 48,6 & 16 & 69,6 & $-1,64$ & 0,05 \\
Casada & 15 & 42,9 & & & - & - \\
Divorciada & 2 & 5,7 & 1 & 4,3 & - & - \\
Conviviente & 1 & 2,9 & 6 & 26,1 & - & \\
\hline Total & 35 & 100,0 & 23 & 100,0 & & \\
\hline
\end{tabular}

Respecto a la utilización de método anticonceptivo, este fue utilizado por el $73,9 \%$ de las trabajadoras sexuales y el $65,7 \%$ del grupo control, diferencia no significativa $(p=0,250)$. Utilizan algún método anticonceptivo el $73,9 \%$ de las trabajadoras sexuales y el $65,7 \%$ del grupo control, diferencia no significativa $(p=0,250)$. Sin embargo, respecto del tipo de método anticonceptivo utilizado en ambos grupos, se observaron diferencias estadísticamente significativas. El tratamiento hormonal $(45,7 \%)$ fue el preferido por el grupo control y el dispositivo intrauterino $(30,4 \%)$ en el grupo de trabajadoras sexuales (Tabla III).
Consultadas sobre abusos sexuales, el $100 \%$ de las mujeres del grupo control no había sido abusada, a diferencia del $30,4 \%$ de las trabajadoras sexuales; de este porcentaje, un $21,5 \%$ eran solteras en el momento de ocurrido el hecho y el $8,7 \%$ estaban conviviendo (Figura 2).

Respecto de las infecciones de transmisión sexual (ITS) y VIH, en el grupo de las trabajadoras sexuales que conviven se registraron los siguientes hallazgos: de un total de 6 mujeres, el $50,0 \%$ presentó ITS y un $16,7 \% \mathrm{VIH}$. En el grupo control solo se presentó un caso de ITS lo que corresponde al 5,9\% del total de mujeres con dicho estado civil $(n=17)$.

Tabla III

TIPO DE ANTICONCEPTIVO UTILIZADO SEGÚN GRUPO DE MUJERES

\begin{tabular}{|c|c|c|c|c|c|c|c|c|}
\hline \multirow{2}{*}{$\begin{array}{l}\text { Utiliza método } \\
\text { anticonceptivo }\end{array}$} & \multirow[t]{2}{*}{ Método } & \multicolumn{2}{|c|}{ Grupo control } & \multicolumn{2}{|c|}{ Trabajadora Sexual } & \multirow{2}{*}{$\mathrm{Z}_{\mathrm{C}}$} & \multirow{2}{*}{$\mathrm{p}$} & \\
\hline & & $\mathrm{n}$ & $\%$ & $n$ & $\%$ & & & \\
\hline \multirow[t]{4}{*}{$\mathrm{Si}$} & DIU & 2 & 5,7 & 7 & 30,4 & -2.38 & 0,009 & $s$ \\
\hline & Hormonal & 16 & 45,7 & 5 & 21,7 & 1,99 & 0,023 & $s$ \\
\hline & Otro & 5 & 14,3 & 5 & 21,7 & $-0,71$ & 0,238 & ns \\
\hline & Subtotal & 23 & 65,7 & 17 & 73,9 & 0,67 & 0,250 & ns \\
\hline No & No usa & 12 & 34,3 & 6 & 26,1 & & & \\
\hline Total & Total & 35 & 100,0 & 23 & 100,0 & & & \\
\hline
\end{tabular}




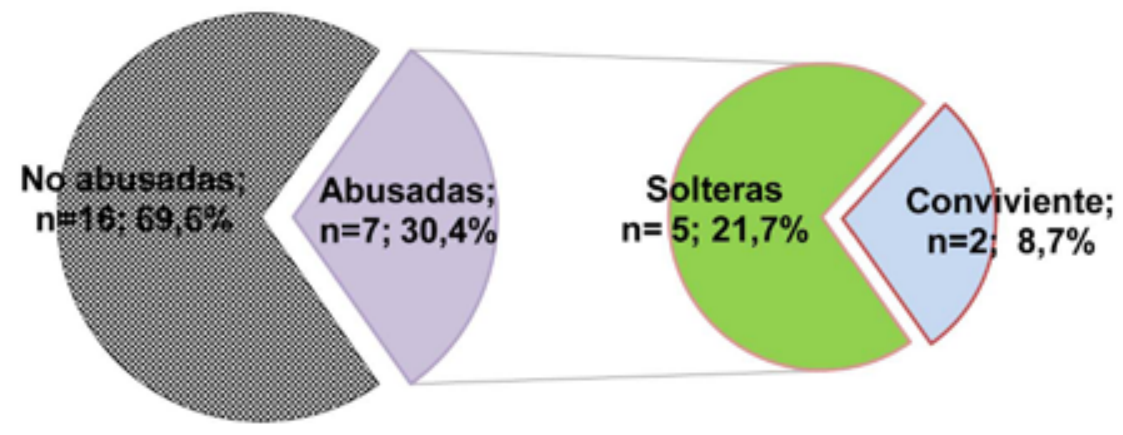

Figura 2. Distribución de mujeres trabajadoras sexuales, que fueron abusadas sexualmente.

Al considerar la variable número de partos entre ambos grupo se pudo determinar que la categoría multíparas es la con mayor porcentaje en ambos grupos con un $45,7 \%$ en el grupo control y un $56,5 \%$ en el grupo de trabajadoras sexuales, diferencias estadísticamente no significativas (Tabla IV).

Consultados sobre la frecuencia sexual con una pareja sentimentalmente comprometida, la respuesta más recurrente fue 1 a 2 veces por semana, representando un $67,6 \%$ en el caso de las mujeres del grupo control y un $39,1 \%$ del grupo trabajadoras sexuales. No obstante en este último grupo, el $21,7 \%$ de las solteras indicó una frecuencia entre 5 y 8 por semana, a diferencias de un $2,9 \%$ del grupo control (Tabla V).

Tabla IV

PARIDAD SEGÚN GRUPO

\begin{tabular}{cccccccc}
\hline Número de partos & \multicolumn{2}{c}{ Grupo control } & \multicolumn{2}{c}{ Trabajadora Sexual } & \multirow{2}{*}{$\mathrm{Z}_{\mathrm{C}}$} & $\mathrm{p}$ & \\
\cline { 2 - 5 } & $\mathrm{n}$ & $\%$ & $\mathrm{n}$ & $\%$ & & & \\
\hline Nulíparas & 10 & 28,6 & 4 & 17,4 & 1,02 & 0,846 & $\mathrm{~ns}$ \\
Primíparas & 9 & 25,7 & 6 & 26,1 & $-0,03$ & 0,487 & $\mathrm{~ns}$ \\
Multíparas & 16 & 45,7 & 13 & 56,5 & $-0,81$ & 0,209 & $\mathrm{~ns}$ \\
\hline Total & 35 & 100,0 & 23 & 100,0 & & & \\
\hline
\end{tabular}

Tabla V

FRECUENCIA DE RELACIÓN SEXUAL SEMANAL DE LAS MUJERES SENTIMENTALMENTE COMPROMETIDAS SEGÚN GRUPO

\begin{tabular}{|c|c|c|c|c|c|}
\hline \multirow[b]{2}{*}{ Calidad } & \multirow[b]{2}{*}{ Frecuencia } & \multicolumn{2}{|c|}{ Estado Civil } & \multirow[b]{2}{*}{ Total } & \multirow[b]{2}{*}{$\%$} \\
\hline & & Soltera & No Soltera & & \\
\hline \multirow{5}{*}{ Grupo control } & $<1$ & 0 & 3 & 3 & 8,8 \\
\hline & $1-2$ & 9 & 14 & 23 & 67,6 \\
\hline & $3-5$ & 6 & 1 & 7 & 20,6 \\
\hline & $5-8$ & 1 & 0 & 1 & 2,9 \\
\hline & Subtotal & 16 & 18 & 34 & 100,0 \\
\hline \multirow{5}{*}{ Trabajadora Sexual } & $<1$ & 2 & 3 & 5 & 21,7 \\
\hline & $1-2$ & 7 & 2 & 9 & 39,1 \\
\hline & $3-5$ & 3 & 1 & 4 & 17,4 \\
\hline & $5-8$ & 4 & 1 & 5 & 21,7 \\
\hline & Subtotal & 16 & 7 & 23 & 100,0 \\
\hline
\end{tabular}


Descritas las características sociodemográficas y comportamiento sexual de los grupos estudiados, se procedió a calcular los puntajes del IFSF en general y en las distintas dimensiones. Al analizar este índice en cada grupo, respecto a sus parejas sentimentalmente comprometidas, ninguno de ellos presentó diferencias estadísticamente significativas, a pesar de observarse mayores valores en las trabajadoras sexuales, por ejemplo en excitación (4,3 puntos) y lubricación (5,1 puntos), por sobre 3,9 y 4,7 puntos del grupo control (Tabla VI, Figura 3 y 4 ).

\section{Tabla VI}

PUNTAJES DEL ÍNDICE DE FUNCIÓN SEXUAL FEMENINA DE LAS MUJERES SENTIMENTALMENTE COMPROMETIDAS SEGÚN GRUPO

\begin{tabular}{|c|c|c|c|c|c|c|}
\hline Calidad & Estadísticas & $\begin{array}{l}\text { Grupo } \\
\text { Control }\end{array}$ & $\begin{array}{c}\text { Trabajadora } \\
\text { sexual }\end{array}$ & $z_{c}$ & $\mathrm{p}$ & \\
\hline \multirow{5}{*}{$\begin{array}{l}\text { Índice de } \\
\text { Función Sexual }\end{array}$} & Media & 25,0 & 25,5 & $-0,59$ & 0,556 & ns \\
\hline & Mediana & 27,0 & 26,7 & & & \\
\hline & D. Estándar & 7,0 & 6,7 & & & \\
\hline & Mínimo & 3,6 & 12,1 & & & \\
\hline & Máximo & 32,9 & 32,6 & & & \\
\hline \multirow[t]{5}{*}{ Deseo } & Media & 3,6 & 3,6 & $-0,24$ & 0,808 & ns \\
\hline & Mediana & 3,6 & 3,6 & & & \\
\hline & D. Estándar & 0,9 & 1,5 & & & \\
\hline & Mínimo & 1,8 & 1,2 & & & \\
\hline & Máximo & 5,4 & 6,0 & & & \\
\hline \multirow[t]{5}{*}{ Excitación } & Media & 3,9 & 4,3 & $-1,45$ & 0,146 & ns \\
\hline & Mediana & 4,2 & 5,1 & & & \\
\hline & D. Estándar & 1,5 & 1,7 & & & \\
\hline & Mínimo & 0,0 & 1,2 & & & \\
\hline & Máximo & 6,0 & 6,0 & & & \\
\hline \multirow[t]{5}{*}{ Lubricación } & Media & 4,7 & 5,1 & $-0,91$ & 0,363 & ns \\
\hline & Mediana & 5,1 & 5,4 & & & \\
\hline & D. Estándar & 1,6 & 1,3 & & & \\
\hline & Mínimo & 0,0 & 1,2 & & & \\
\hline & Máximo & 6,0 & 6,0 & & & \\
\hline \multirow[t]{5}{*}{ Orgasmo } & Media & 3,4 & 3,6 & $-1,03$ & 0,302 & ns \\
\hline & Mediana & 3,9 & 4,2 & & & \\
\hline & D. Estándar & 1,4 & 1,3 & & & \\
\hline & Mínimo & 0,0 & 0,9 & & & \\
\hline & Máximo & 4,5 & 4,5 & & & \\
\hline \multirow[t]{5}{*}{ Satisfacción } & Media & 4,4 & 4,6 & $-0,92$ & 0,357 & ns \\
\hline & Mediana & 5,2 & 5,6 & & & \\
\hline & D. Estándar & 1,7 & 1,6 & & & \\
\hline & Mínimo & 0,0 & 1,6 & & & \\
\hline & Máximo & 6,0 & 6,0 & & & \\
\hline \multirow[t]{5}{*}{ Dolor } & Media & 5,0 & 4,3 & $-1,51$ & 0,132 & ns \\
\hline & Mediana & 6,0 & 4,4 & & & \\
\hline & D. Estándar & 1,6 & 1,9 & & & \\
\hline & Mínimo & 0,0 & 0,0 & & & \\
\hline & Máximo & 6,0 & 6,0 & & & \\
\hline
\end{tabular}




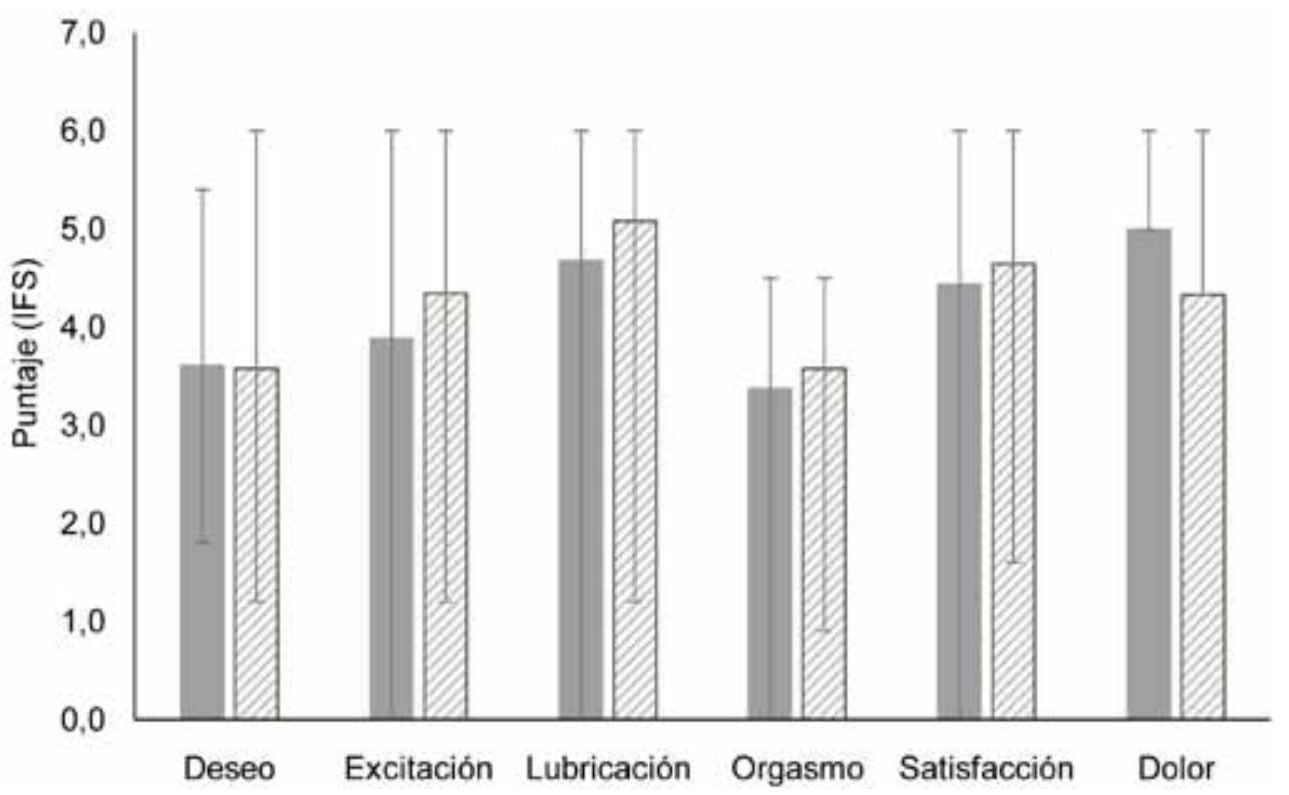

Figura 3. Estadísticas puntajes IFS femenina según dimensión (promedio, máximo y mínimo).

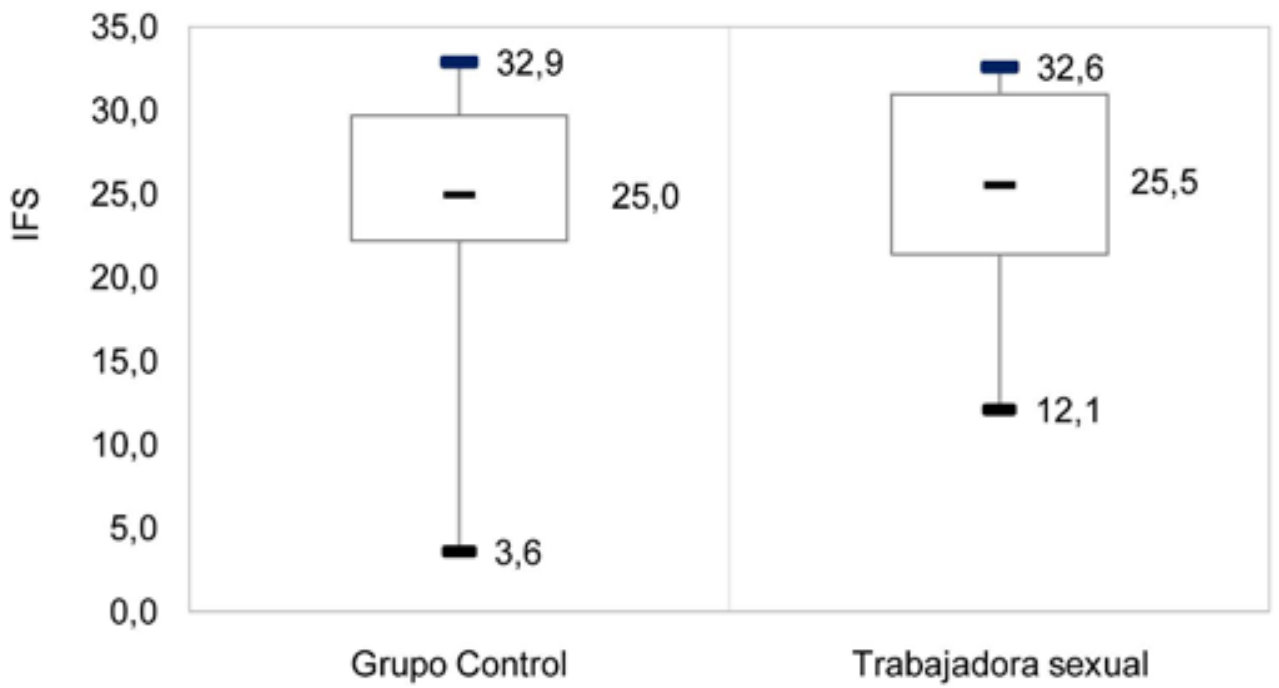

Figura 4. Gráfico de cajas para puntajes IFS femenina según grupo de estudio. 


\section{DISCUSIÓN}

Nuestros resultados muestran que no hubo diferencias significativas en el IFSF entre trabajadoras sexuales y el grupo control. Estudios internacionales han analizado a las trabajadoras sexuales pero en temas relacionados con el ambiente familiar que las rodea (14), infecciones de transmisión sexual (15) y alteraciones en la calidad de vida sexual debido a esas patología (16), sin embargo, no hemos encontrado estudios que analicen el IFSF de manera comparativa.

Algunas investigaciones han establecido que las trabajadoras sexuales provienen de un nivel socioeconómico bajo, que han sufrido de abusos sexuales durante la niñez, que abandonan el hogar parental tempranamente y no completan estudios terciarios (14). Debido a lo anterior y para contextualizar los resultados obtenidos en el presente estudio, comparamos previamente algunas características sociodemográficas de ambos grupos, entre ellas: edad, nivel educacional, estado civil, método anticonceptivo y presencia de abuso sexual. Con respecto a esto, Silva-Camara y cols (17), han determinado que mujeres menores de 40 años presentan puntuaciones IFSF significativamente más altas, con mayores niveles de deseo, excitación, lubricación y satisfacción que las mujeres mayores de 40 años, las que presentan un mayor riesgo de experimentar disfunción sexual (17). Sin embargo, en nuestro estudio, ambos grupos presentaron una edad promedio similar, que fue inferior a los 40 años, sin presentar diferencias significativas $(p=0,20)$.

Otros estudios señalan que el nivel educacional influye sobre el comportamiento sexual (18). En este estudio si bien las diferencias observadas en nivel educacional son importantes e inversas (al comparar la educación básica y superior entre trabajadoras sexuales y el grupo control), no se pudo determinar diferencias significativas, debidas principalmente al bajo tamaño muestral y esta es una debilidad de nuestra investigación.

En nuestro estudio las trabajadoras sexuales son principalmente solteras, a diferencias del grupo control, diferencia significativa $(p=0,05)$. Estos datos al asociarlo con estudios realizados harían suponer que aumentaría el riesgo de disfunción sexual, puesto que tener una pareja estable, es un factor protector del riesgo de disfunción sexual $(18,19)$. No obstante, otro estudio ha determinado que mujeres que llevan menos tiempo casada presentan un IFSF mayor, que aquellas con un tiempo prolongado de matrimonio (17).

También se evaluó el tipo de método anticonceptivo utilizado, siendo el tratamiento hormonal el preferido por las mujeres del grupo control y el DIU por las trabajadoras sexuales. Respecto de lo anterior, por muchos años se ha considerado que la utilización del DIU afectaría a la mujer nulípara que planea tener hijos, esto debido a una mayor tendencia a la enfermedad inflamatoria pélvica (20). Por lo tanto, una de las posibles explicaciones para la mayor utilización de DIU sería un mayor número de partos en el grupo de trabajadoras sexuales o que existiera un número considerable de nulíparas en el grupo control. Sin embargo, no se observaron diferencias significativas entre ambos grupos. Otra explicación posible se relaciona con el desempeño de las trabajadoras sexuales, el cual está asociado al consumo de alcohol. Por lo tanto, se puede sugerir que el grupo de trabajadoras sexuales, prefiere el método del DIU, debido a que el alcohol eventualmente interactúa con el tratamiento hormonal (21). Anteriormente, se ha determinado que la utilización o no de un método anticonceptivo no influye en el IFSF (22). Sin embargo, se ha publicado recientemente que la utilización del DIU aumenta la prevalencia de disfunción sexual (23).

Respecto al antecedente de abuso sexual, este sólo se presentó en el grupo de trabajadoras sexuales. Se ha descrito que las mujeres abusadas durante la infancia, tienden a presentar en la adultez dificultades en el ámbito sexual (24).

Finalmente, en este estudio no se encontraron diferencias significativas en el IFSF entre el grupo control y el grupo de trabajadoras sexuales con 25,0 y 25,5 puntos respectivamente; media que se ubica dentro de los niveles reportados internacionalmente para la población general $(25,26)$, pero por debajo de lo estimado en estudios nacionales (27) y por sobre lo descrito en temas relacionados con el trastorno de la excitación sexual femenina (12).

\section{CONCLUSIÓN}

A pesar de que varias de las características socio-demográficas (nivel educacional, estado civil, DIU y abuso sexual) presentes en el grupo de trabajadoras sexuales representan un mayor riesgo de disfunción sexual, las mujeres que se desempeñan en el comercio sexual no presentaron diferencias significativas en el IFSF respecto a las mujeres del grupo control. Finalmente, existe muy poca literatura que evalué la función sexual en las mujeres se desempeñan como trabajadoras sexuales, aportando con este estudio una primera mirada, que puede ayudar a proponer nuevos enfoques e investigaciones en esta área.

\section{REFERENCIAS}

1. Aggleton P, Parker R. Routledge, Editores, Handbook of Sexuality, Health and Rights. UK: Taylor and Francis group; 2010, capítulo 15.

2. Charnay M, Henríquez E. Prevalence of inhibited sexual drive in women actively reproductive and related factors. Ciencia y Enfermeria 2003;IX(1):55-64. 
3. Hohmann G, Mundry R, Deschner T. The relationship between socio-sexual behavior and salivary cortisol in bonobos: tests of the tension regulation hypothesis. Am J Primatol 2009;71(3):223-32.

4. Zeidenstein S, Moore K. Aprendiendo sobre sexualidad. The Population Council. Nueva York. 1999.

5. Ewing ET. Maternity and modernity: Soviet women teachers and the contradictions of Stalinism. Womens Hist Rev 2010;19(3):451-7.

6. Blümel JE, Araya $H$, Riquelme R, Castro G, Sánchez F, Gramegna G. Prevalence of sexual dysfunction in climacteric women. Influence of menopause and hormone replace therapy. Rev Med Chil 2002;130(10):1131-8

7. Masters W, Johnson V. Respuesta Sexual Humana. Buenos Aires: Editorial Intermédica. 1967.

8. Castro R, González M, Lopez C, Editores. Manual de atención personalizada en el proceso reproductivo. MINSAL 2008.

9. Salonia A, Giraldi A, Chivers ML, Georgiadis JR, Levin R, Maravilla KR, McCarthy MM. Physiology of women's sexual function: basic knowledge and new findings. Sex Med 2010;7(8):2637-60.

10. Puppo V. Embryology and anatomy of the vulva: the female orgasm and women's sexual health. Eur $J$ Obstet Gynecol Reprod Biol 2011;154(1):3-8.

11. Lewis R. The International Society for Sexual Medicine: A Rich History and a Bright Future. Sexual Medicine Reviews 2013;1(2):65-75.

12. Rosen R, Brown C, Heiman J, Leiblum S, Meston C, Shabsigh R, Ferguson D, D'agostino R. The Female Sexual Function Index (FSFI): A Multidimensional Self-Report Instrument for the Assessment of Female Sexual Function. J Sex Marital Ther 2000;26(2):191208.

13. Blümel J, Bravo F, Recavarren M, Sarrá S. Función sexual en mujeres usuarias de terapia de reemplazo hormonal. Rev Méd Chile 2003;131(11):1251-5.

14. Potter K, Martin J, Romans S. Early developmental experiences of female sex workers: a comparative study. Aust N Z J Psychiatry 1999;33(6):935-40.

15. Gezie LD, Taye BW, Ayele TA. Time to unsafe sexual practice among cross-border female sex workers in Metemma Yohannes, North West Ethiopia. BMC Public Health 2015; 28:15:710.
16. Bruce E, Bauai L, Masta A, Rooney PJ, Paniu M, Sapuri M, Keogh L, Kaldor J, Fairley CK. A crosssectional study of reported symptoms for sexually transmissible infections among female sex workers in Papua New Guinea. Sex Health 2010;7(1):71-6.

17. Silva Camara $C$, Veloso Corrêa $H$, Brito e Silva $S$, Alves da Silva C, Silva Junior M, Brito R. Life cycle comparative analysis of sexual function in women with normal and overweight body mass index. Creative Education 2014;5:1363-76.

18. Blümel JE, Binfa L, Cataldo $P$, Carrasco A, Izaguirre $\mathrm{H}$, Sarrá $\mathrm{S}$. Índice de función sexual femenina: un test para evaluar la sexualidad de la mujer. Rev Chil Obstet Ginecol 2004;69(2):118-25.

19. Laumann EO, Paik A, Rosen RC. Sexual Dysfunction in the United States. prevalence and predictors. JAMA 1999;281(6):537-44.

20. Daling JR, Weiss NS, Metch BJ, Chow WH, Soderstrom RM, Moore DE, Spadoni LR, Stadel BV. Primary tubal infertility in relation to the use of an intrauterine device. N Engl J Med 1985;312(15):937-41.

21. Jones MK, Jones BM. Ethanol metabolism in women taking oral contraceptives. Alcohol Clin Exp Res 1984;8(1):24-8.

22. Jaafarpour M, Khani A, Khajavikhan J, Suhrabi Z. Female sexual dysfunction: prevalence and risk factors. $\mathrm{J}$ Clin Diagn Res 2013;7(12):2877-80.

23. Panchalee $\mathrm{T}$, Wongwananuruk $\mathrm{T}$, Augsuwatana $\mathrm{S}$, Sirimai K, Tammakunto M, Neangton C, Bunsuk S, Inthawong $\mathrm{J}$. Prevalence and associating factors of sexual dysfunction in women who use intrauterine device (IUD) for contraception. J Med Assoc Thai 2014;97(1):20-7.

24. Stephenson KR, Pulverman CS, Meston CM. Assessing the association between childhood sexual abuse and adult sexual experiences in women with sexual difficulties. J Trauma Stress 2014;27(3):274-82.

25. Ghassamia M, Asghari A, Shaeiri MR, Safarinejad MR. Validation of psychometric properties of the Persian version of the Female Sexual Function Index. Urol J 2013;10(2):878-85.

26. Crisp C, Fellner A, Pauls R. Validation of the Female Sexual Function Index (FSFI) for web-based administration. Int Urogynecol J2015;26(2):219-22.

27. Valenzuela R, Contreras $Y$, Manriquez K. Índice de función sexual en trabajadoras de la salud. Rev Chil Obstet Ginecol 2014;79(2):92-101. 